\title{
Thermo Elastic-Plastic Analysis of Rotating Functionally Graded Stainless Steel Composite Cylinder under Internal and External Pressure Using Finite Difference Method
}

\author{
Sanjeev Sharma and Sanehlata Yadav \\ Department of Mathematics, Jaypee Institute of Information Technology, A-10, Sector 62, Noida, Uttar Pradesh 201307, India \\ Correspondence should be addressed to Sanjeev Sharma; sanjeev.sharma@jiit.ac.in
}

Received 22 July 2013; Accepted 26 October 2013

Academic Editor: Mohd Sapuan Salit

Copyright (C) 2013 S. Sharma and S. Yadav. This is an open access article distributed under the Creative Commons Attribution License, which permits unrestricted use, distribution, and reproduction in any medium, provided the original work is properly cited.

\begin{abstract}
The thermal elastic-plastic stresses have been investigated for a rotating functionally graded stainless steel composite cylinder under internal and external pressure with general nonlinear strain hardening law and von Mises' yield criterion using finite difference method. The modulus of elasticity in the rotating cylinder varies radially according to power law and the temperature distribution satisfies Laplace heat equation in radial direction. From the analysis, we can conclude that cylinder made of functionally graded stainless steel composite material with variable thickness and variable density under thermal loading for Swift's strain hardening measure $m=0.6$ is better choice of the design as compared to homogeneous cylinder. This is because of the reason that circumferential stress is less for functionally graded stainless steel composite cylinder as compared to homogeneous cylinder for Swift's strain hardening measure $m=0.6$ under internal and external pressure. This leads to the idea of "stress saving" which minimizes the possibility of fracture of cylinder.
\end{abstract}

\section{Introduction}

Thermo elastic-plastic analysis of functionally graded materials under internal and external pressure is an active topic for engineering mechanics. The study of thick-walled cylinder is an interesting area of research and highly used due to vast utilization in the pressure vessels and pipes and so forth. Functionally graded materials are nothing but nonhomogeneous composite materials which are highly heat resistant and very promising in high-tech engineering fields $[1,2]$. The demands of functionally graded materials are increasing under high rotation and temperature. These materials find their applications in many areas, that is, flywheels, aerospace, nuclear reactors, compressors, and so forth. The problems of rotating thick-walled cylinder and plates can be found in many text books of elasticity $[3,4]$. Obata et al. [5] calculated stresses for a hollow circular cylinder and hollow sphere under thermal loading using perturbation method. For these materials they found that the perturbation solutions had good convergence. Perry and Aboudi [6] calculated the residual stresses in a homogeneous cylinder using finite difference method and concluded that it is very effective in solving autofrettage problem numerically. Gao [7] investigated elastic-plastic stresses, strains, and displacements in a cylinder under internal pressure using elastic strain hardening in the plane strain conditions. Tutuncu and Ozturk [8] studied the closed form solution for stresses and displacement in cylindrical and spherical vessels made of functionally graded material subjected to internal pressure using infinitesimal theory of elasticity. They compared stress distributions in functionally graded material with homogeneous material. Singh and Gupta [9] developed a mathematical model to describe steady-state creep in an isotropic functionally graded composite subjected to internal pressure which contains linearly varying silicon carbide particles in a matrix of pure aluminum. They observed that the radial stress in the cylinder decreases throughout with the increase in reinforcement gradient, whereas the tangential, 
axial, and effective stresses increase significantly near the inner radius but show significant decrease towards the outer radius. Aggarwal et al. [10] investigated safety factors in terms of elastic-plastic stresses for functionally graded thickwalled circular cylinder under internal and external pressure and concluded that functionally graded thick-walled cylinder minimizes the possibility of fracture of the cylinder. Aggarwal et al. [11] calculated thermal creep stresses for nonhomogeneous thick-walled cylinder under internal and external pressure using Lebesgue strain measure and concluded that nonhomogeneous cylinder is better choice of design as compared to homogeneous cylinder. Parvizi et al. [12] studied a mathematical model to predict the yielding in a functionally graded $\mathrm{Al} \mathrm{A} 359 / \mathrm{SiCp}$ cylinder and find closed form solution for plastic stresses using Tresca's yield criterion subjected to internal pressure with thermal loading. They observed that there is a point in cylinder where the hoop stress changes from compressive to tensile and the position of this point is independent of the temperature gradient and depends on material properties and geometry of the functionally graded cylinder. Eraslan and Akgül [13] calculated the numerical solution for elastic-plastic stresses in a rotating disk with von Mises' yield criterion using general nonlinear strain hardening rule.

In this paper, thermal elastic-plastic stresses have been calculated for cylinder made up of functionally graded stainless steel composite material under internal and external pressure using finite difference method. In this problem, a general nonlinear strain hardening law with von Mises' yield criterion has been considered. Results have been discussed numerically with the help of graphs and tables.

\section{Objective}

For a rotating cylinder with varying material properties, circumferential stress at the hub does not exceed the allowable value which tells the designers little more than that the design of the cylinder is safe at the given pressure. Thus our prime objective is to calculate allowable thermal elasticplastic stresses in an open ended functionally graded stainless steel composite rotating cylinder under pressure for varying expansion to incorporate a "safety factor" that prevents the cylinder from bursting under pressure and thermal loading.

\section{Mathematical Formulation}

3.1. Distribution of Material Properties. Consider a long open ended axisymmetric cylinder made up of functionally graded stainless steel composite material with inner and outer radii $a$ and $b$, respectively, and the cylinder is subjected to internal pressure $p_{i}$ and external pressure $p_{0}$ as shown in Figure 1. The cylindrical polar coordinates $(r, \theta)$ under plane stress condition have been considered in this problem.

In this study, Poisson's ratio $(\nu)$ and thermal expansion coefficient $\left(\alpha=\alpha_{0}\right)$ are assumed to be constants. The other properties, that is, Young's modulus which is defined by power law, temperature distribution that follows Laplace

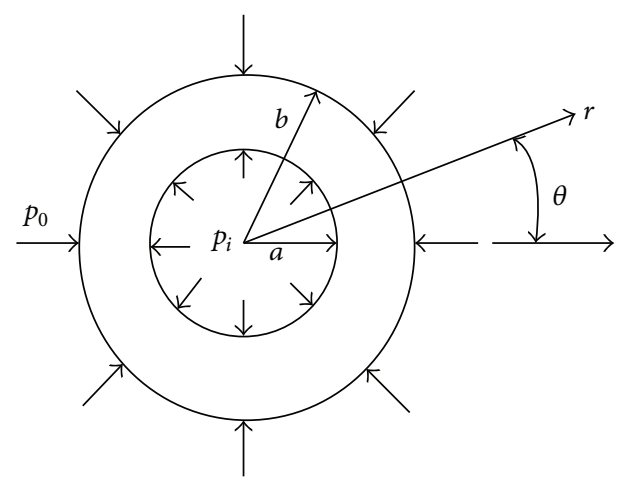

FIgURE 1: A functionally graded rotating cylinder with inner pressure $p_{i}$ and outer pressure $p_{0}$ at the boundary.

heat equation in radial direction, thickness, and density, are varying radially and are expressed as

$$
\begin{array}{ll}
E(r)=E_{0}\left(\frac{r}{b}\right)^{e_{1}}, & \theta(r)=\overline{\theta_{0}} \log \left(\frac{r}{b}\right), \\
\rho(r)=\rho_{0}\left(\frac{r}{b}\right)^{d}, & h(r)=h_{0}\left(\frac{r}{b}\right)^{-l},
\end{array}
$$

where $\overline{\theta_{0}}=\theta_{0} /(\log (a / b)), r$ is the radius of the cylinder, $E_{0}$, $\rho_{0}, \theta_{0}$, and $h_{0}$ are material constants, and $e_{1}, d$, and $l$ are the geometric parameters.

3.2. Basic Equations. The equilibrium equation for the cylinder in the absence of body forces is

$$
\frac{d}{d r}\left(h r T_{r r}\right)-h T_{\theta \theta}+h \rho \omega^{2} r^{2}=0,
$$

where $T_{r r}$ and $T_{\theta \theta}$ are radial and circumferential stresses, respectively.

Using infinitesimal theory of elasticity, the relations between strains and radial displacements are

$$
e_{r}=\frac{d u}{d r}, \quad e_{\theta}=\frac{u}{r}
$$

where $e_{r}$ and $e_{\theta}$ are radial and circumferential strains, respectively, and $u$ is the radial displacement.

The equation of compatibility can be derived from (3) as

$$
\frac{d e_{\theta}}{d r}+\frac{e_{\theta}-e_{r}}{r}=0
$$

From infinitesimal theory of elasticity, the stress-strain relations are

$$
\begin{gathered}
e_{r}^{e}=\frac{1}{E}\left[T_{r r}-v\left(T_{\theta \theta}+T_{z z}\right)\right], \quad e_{\theta}^{e}=\frac{1}{E}\left[T_{\theta \theta}-\nu\left(T_{r r}+T_{z z}\right)\right], \\
e_{z}^{e}=\frac{1}{E}\left[T_{z z}-v\left(T_{r r}+T_{\theta \theta}\right)\right],
\end{gathered}
$$

where $e_{r}^{e}, e_{\theta}^{e}$, and $e_{z}^{e}$ are the elastic radial, circumferential, and axial strains, respectively. 
Due to geometric symmetry of the cylinder, circumferential displacement, shear stresses, and strains are assumed to be zero.

Using deformation theory of plasticity, the relation between the stresses and plastic strains can be determined as

$$
\begin{aligned}
& e_{r}^{p}=\frac{e_{e}^{p}}{T_{e e}}\left[T_{r r}-\frac{1}{2}\left(T_{\theta \theta}+T_{z z}\right)\right], \\
& e_{\theta}^{p}=\frac{e_{e}^{p}}{T_{e e}}\left[T_{\theta \theta}-\frac{1}{2}\left(T_{r r}+T_{z z}\right)\right], \\
& e_{z}^{p}=\frac{e_{e}^{p}}{T_{e e}}\left[T_{z z}-\frac{1}{2}\left(T_{r r}+T_{\theta \theta}\right)\right],
\end{aligned}
$$

where $T_{e e}$ is the equivalent stress, $e_{e}^{p}$ is the equivalent plastic strain, and $e_{r}^{p}, e_{\theta}^{p}$, and $e_{z}^{p}$ are the plastic radial, circumferential, and axial strains, respectively.

von Mises' yield criterion is given by

$$
T_{e e}=\sqrt{\left(T_{r r}-T_{\theta \theta}\right)^{2}+\left(T_{\theta \theta}-T_{z z}\right)^{2}+\left(T_{z z}-T_{r r}\right)^{2}} .
$$

The total radial, circumferential, and axial strains in thick-walled rotating cylinder are

$$
\begin{gathered}
e_{r}=e_{r}^{e}+e_{r}^{p}+\alpha \theta, \quad e_{\theta}=e_{\theta}^{e}+e_{\theta}^{p}+\alpha \theta \\
e_{z}=e_{z}^{e}+e_{z}^{p}+\alpha \theta
\end{gathered}
$$

The temperature field satisfying Laplace heat equation is

$$
\frac{d^{2} \theta}{d r^{2}}+\frac{1}{r} \frac{d \theta}{d r}=0
$$

with $\theta=\theta_{0}$ at $r=a$ and $\theta=0$ at $r=b$, where $\theta_{0}$ is a constant, given by $\theta(r)=\overline{\theta_{0}} \log (r / b)$.

We define the stress function $\phi(r)$ for thick-walled rotating cylinder which is related to radial and hoop stresses as

$$
T_{r r}=\frac{\phi}{h r}, \quad T_{\theta \theta}=\frac{1}{h} \frac{d \phi}{d r}+\rho \omega^{2} r^{2}, \quad T_{z z}=0 .
$$

Since it has been assumed that the cylinder is long and open ended and there is plane stress condition, therefore axial stress is zero; that is, $T_{z z}=0$.
Substituting (10) and (5) into (8), we have

$$
\begin{aligned}
& e_{r}=\frac{1}{E}\left(\frac{\phi}{h r}-\nu \frac{1}{h} \frac{d \phi}{d r}-v \rho \omega^{2} r^{2}\right)+e_{r}^{p}+\alpha \theta, \\
& e_{\theta}=\frac{1}{E}\left(\frac{1}{h} \frac{d \phi}{d r}+\rho \omega^{2} r^{2}-\nu \frac{\phi}{h r}\right)+e_{\theta}^{p}+\alpha \theta, \\
& e_{z}=\frac{-v}{E}\left(\frac{1}{h} \frac{d \phi}{d r}+\rho \omega^{2} r^{2}+\frac{\phi}{h r}\right)+e_{z}^{p}+\alpha \theta .
\end{aligned}
$$

Substituting of (11) into compatibility (4), we have

$$
\begin{aligned}
r^{2} \phi^{\prime \prime} & -r \phi^{\prime}\left[1+r\left(\frac{h^{\prime}}{h}+\frac{E^{\prime}}{E}\right)\right]-\phi\left[1-r \nu\left(\frac{h^{\prime}}{h}+\frac{E^{\prime}}{E}\right)\right] \\
& +h \rho^{\prime} \omega^{2} r^{4}+\left(3+\nu-r \frac{E^{\prime}}{E}\right) h \rho \omega^{2} r^{3} \\
& -E h r\left(e_{r}^{p}-e_{\theta}^{p}\right)+E h r^{2}\left[\left(e_{\theta}^{p}\right)^{\prime}+\alpha \theta^{\prime}+\alpha^{\prime} \theta\right]=0,
\end{aligned}
$$

where $\phi^{\prime}=d \phi / d r, \phi^{\prime \prime}=d^{2} \phi / d r^{2}$, and $e_{\theta}^{p^{\prime}}=d e_{\theta}^{p} / d r$.

The relation between the yield stress $T_{e e}$ and the equivalent plastic strain $e_{e}^{p}$ for Swift's hardening law can be expressed as

$$
\begin{gathered}
e_{e}^{e}=\frac{T_{e e}}{E}, \quad e_{e} \leq e_{0}, \\
e_{e}^{p}=\frac{1}{\eta}\left[\left(\frac{T_{e e}}{T_{0}}\right)^{m}-1\right], \quad e_{e}>e_{0},
\end{gathered}
$$

where $\eta, m, T_{0}, e_{e}$, and $e_{0}$ are hardening parameter, material parameter, yield limit, equivalent total strain, and yield strain, respectively.

Substituting $e_{e}^{p}$ from (13) into (6) results in

$$
\begin{aligned}
& e_{r}^{p}=\frac{(1 / \eta)\left[\left(T_{e e} / T_{0}\right)^{m}-1\right]}{T_{e e}}\left[T_{r r}-0.5\left(T_{\theta \theta}+T_{z z}\right)\right], \\
& e_{\theta}^{p}=\frac{(1 / \eta)\left[\left(T_{e e} / T_{0}\right)^{m}-1\right]}{T_{e e}}\left[T_{\theta \theta}-0.5\left(T_{r r}+T_{z z}\right)\right], \\
& e_{z}^{p}=\frac{(1 / \eta)\left[\left(T_{e e} / T_{0}\right)^{m}-1\right]}{T_{e e}}\left[T_{z z}-0.5\left(T_{r r}+T_{\theta \theta}\right)\right] .
\end{aligned}
$$

Substituting (14) into (12), we have

$$
\left.\left.\begin{array}{rl}
r^{2} \phi^{\prime \prime} & {\left[1+\frac{E}{2 \eta T_{e e}^{2}}\left\{2 T_{e e}\left[\left(\frac{T_{e e}}{T_{0}}\right)^{m}-1\right]+\frac{1}{2 T_{e e}}\left(T_{r r}-2 T_{\theta \theta}\right)^{2} \times\left(1+(m-1)\left(\frac{T_{e e}}{T_{0}}\right)^{m}\right)\right\}\right]} \\
& +E h r^{2}\left[\theta \alpha^{\prime}+\alpha \theta^{\prime}\right]+h \rho \omega^{2} r^{3}(E+4 v) \\
& -\frac{E}{2 \eta T_{e e}^{2}}\left[+\frac{1}{2 T_{e e}}\left(1+(m-1)\left(\frac{T_{e e}}{T_{0}}\right)^{m}\right)\left(T_{r r}-2 T_{\theta \theta}\right) \times\left\{\left(\frac{T_{e e}}{T_{0}}\right)^{m}-1\right] \times\left\{\left(1+2 r \frac{h^{\prime}}{h}\right) r \phi^{\prime}-\left(1+r \frac{h^{\prime}}{h}\right) \phi-2 h \rho^{\prime} \omega^{2} r^{4}-4 h \rho \omega^{2} r^{3}\right\}\right. \\
+\left(2 T_{r r}-T_{\theta \theta}\right) \times\left[r \phi^{\prime}-\left(1+r \frac{h^{\prime}}{h}\right)\right. & \left(2 T_{\theta \theta}-T_{r r}\right)
\end{array}\right]\right\}
$$




$$
\begin{aligned}
& -\frac{3}{2 \eta T_{e e}} E h r \times\left[\left(\frac{T_{e e}}{T_{0}}\right)^{m}-1\right] \times\left(T_{r r}-T_{\theta \theta}\right)+r \phi^{\prime}\left[1-r \frac{h^{\prime}}{h}-r\left(\frac{E^{\prime}}{E}\right)\right]-\phi\left[1-\nu r \frac{h^{\prime}}{h}-r\left(\frac{E^{\prime}}{E}\right)\right] \\
& =h \omega^{2} r^{4}\left[\rho\left(\frac{E^{\prime}}{E}\right)-\rho^{\prime}\right] .
\end{aligned}
$$

Equation (15) is the differential equation of the functionally graded stainless steel composite rotating cylinder with nonlinear strain hardening subjected to thermal loading in the plastic region in terms of stresses and stress function.

Equation (15) can be described in the general form in terms of stress function as

$$
\phi^{\prime \prime}=f\left(r, \phi, \phi^{\prime}\right) .
$$

Equation (16) is a nonlinear two point boundary value problem and can be solved numerically, subjected to the boundary conditions

$$
T_{r r}=-p_{i}, \quad \text { at } r=a, \quad T_{r r}=-p_{0}, \quad \text { at } r=b, a>0,
$$

where $a$ and $b$ are the inner and outer radii of the cylinder and $p_{i}$ and $p_{0}$ are internal and external pressures, respectively.

Using finite difference method with central difference in (16), we get the following system of equations:

$$
\frac{\phi_{i+1}-2 \phi_{i}+\phi_{i-1}}{(\Delta r)^{2}}=f\left(r, \phi_{i}, \frac{\phi_{i+1}-\phi_{i-1}}{2 \Delta r}\right), \quad i=2,3, \ldots, n \text {. }
$$

Equation (18) consists of algebraic system of $(n-1)$ equations with the boundary conditions $\phi(a)=-p_{i} h a$ and $\phi(b)=$ $-p_{0} h b$. After solving (18) with boundary conditions we get a stress function $\phi$. Then, the radial and circumferential stresses can be obtained from (10) after substituting the value of stress function $\phi$.

\section{Numerical Discussion}

The properties of a functionally graded stainless steel composite thick-walled rotating cylinder under internal and external pressure $p_{i}=150,300$ and $p_{0}=150,300 \mathrm{MPa}$, respectively, subjected to thermal loading $\left(\theta_{0}=0,400,800\right)$ are defined as follows: the radii of the cylinder are taken as $a=0.1 \mathrm{~m}$ and $b=0.5 \mathrm{~m}$, Poisson's ratio $v=0.3$, Young's modulus $E_{0}=207 \mathrm{GPa}$, and thermal expansion coefficient $\alpha=\alpha_{0}=17.8 \times 10^{-6}{ }^{\circ} \mathrm{C}^{-1}$. The geometric parameters of the cylinder are taken as $e_{1}=0,1,2$ in Young's modulus function and $m=0.4,0.6$ is nonlinear strain hardening measure.

To show the effect of internal and external pressure on a functionally graded stainless steel composite rotating cylinder with strain hardening measure $m=0.4,0.6$ having constant thickness and constant density, Tables 1 and 2 show the circumferential stresses with different parameters of Young's modulus $e_{1}=0,1,2$.

It has been observed from Table 1 that, when external pressure is greater than the internal pressure, circumferential stresses approaches tensile to compressible. Also, these stresses are maximum at external surface for homogeneous as well as functionally graded stainless steel composite cylinder. These stresses are less for functionally graded stainless steel composite cylinder as compared to homogeneous cylinder. As nonhomogeneity changes from $e_{1}=1$ to $e_{1}=2$, circumferential stresses decrease significantly. With the introduction of thermal effects circumferential stresses increase for homogeneous as well as for functionally graded stainless steel composite cylinder $\left(e_{1}=1\right)$ but decrease for functionally graded stainless steel composite cylinder with $e_{1}=2$. It has also been noticed from Table 1 that with the increase in thermal effects these stresses decrease significantly for homogeneous as well as for functionally graded stainless steel composite cylinder and are less for the cylinder with nonhomogeneity parameter $e_{1}=2$. With the increase in angular speed, circumferential stresses increase significantly. When external pressure is less than the internal pressure, circumferential stresses are maximum at internal surface for homogeneous cylinder while maximum at external surface for functionally graded stainless steel composite cylinder and these stresses decreased with the change in nonhomogeneity measure from $e_{1}=1$ to $e_{1}=2$. With the introduction of thermal effects circumferential stresses increase for homogenous as well as for functionally graded stainless steel composite measure $e_{1}=1$ while decrease for functionally graded stainless steel composite cylinder with $e_{1}=2$. With the increase in thermal effects these circumferential stresses decrease significantly while increase with the increase in angular speed. It has been observed from Table 2 that the behavior of homogeneous and functionally graded stainless steel composite cylinder same as discussed in Table 1 but it has been observed that, with increase in strain hardening measure from $m=0.4$ to $m=0.6$, these stresses decrease significantly for functionally graded stainless steel composite cylinder.

Tables 3 and 4 have been made for circumferential stresses in rotating cylinders with variable thickness and variable density with different parameters of Young's modulus $e_{1}=$ $0,1,2$ and strain hardening measure $m=0.4,0.6$.

It has been observed from Table 3 that for cylinder with varying thickness and density, whose external pressure is greater than the internal pressure, circumferential stresses approach from tensile to compressible and are maximum at external surface for homogeneous as well as functionally graded stainless steel composite cylinder. These stresses are less for functionally graded stainless steel composite cylinder as compared to homogeneous cylinder with varying thickness and density as well as cylinder with constant thickness and density. With increase in strain hardening measure from $m=0.4$ to $m=0.6$ these circumferential stresses decrease significantly for functionally graded stainless steel 
TABLE 1: Circumferential stresses for rotating cylinder with constant thickness and constant density, $\omega=300$, 500, nonlinear strain hardening measure $m=0.4$, and different parameters of Young's modulus under internal and external pressure.

\begin{tabular}{|c|c|c|c|c|c|c|}
\hline \multirow[t]{2}{*}{$1 E-3 * T_{\theta \theta} \mathrm{MPa}$} & \multirow[t]{2}{*}{$e_{1}$} & \multicolumn{5}{|c|}{$\begin{array}{c}r \\
m=0.4, \omega=300\end{array}$} \\
\hline & & 0.1 & 0.2 & 0.3 & 0.4 & 0.5 \\
\hline \multirow{3}{*}{$\begin{array}{l}p_{i}=150 \\
p_{0}=300 \\
\theta_{0}=0\end{array}$} & 0 & 8.3454530141 & 3.4064915847 & 0.0677187483 & -3.9596784280 & -8.9602457103 \\
\hline & 1 & 3.3799641785 & 2.7171534961 & 0.8969909345 & -2.6654005915 & -8.5548978826 \\
\hline & 2 & 1.1503024505 & 1.8118357165 & 1.2081310260 & -1.5371690373 & -7.8899736228 \\
\hline \multirow{3}{*}{$\begin{array}{l}p_{i}=300 \\
p_{0}=150 \\
\theta_{0}=0\end{array}$} & 0 & 8.8188655176 & 3.6471590677 & 0.2649872301 & -3.7776104911 & -8.7852151029 \\
\hline & 1 & 3.6049642996 & 2.9421535689 & 1.1219909709 & -2.4404004448 & -8.3298983560 \\
\hline & 2 & 1.1869707579 & 1.9774764390 & 1.4492915544 & -1.2424185539 & -7.5536537230 \\
\hline \multirow{3}{*}{$\begin{array}{l}p_{i}=150 \\
p_{0}=300 \\
\theta_{0}=400\end{array}$} & 0 & 8.3454529134 & 3.4064914474 & 0.0677186071 & -3.9596781512 & -8.9602463330 \\
\hline & 1 & 3.3799641163 & 2.7171535146 & 0.8969910927 & -2.6654006381 & -8.5548985793 \\
\hline & 2 & 1.1503024507 & 1.8118357152 & 1.2081310262 & -1.5371690375 & -7.8899736082 \\
\hline \multirow{3}{*}{$\begin{array}{l}p_{i}=300 \\
p_{0}=150 \\
\theta_{0}=400\end{array}$} & 0 & 8.8188661275 & 3.6471591256 & 0.2649873453 & -3.7776106334 & -8.7852145930 \\
\hline & 1 & 3.6049644528 & 2.9421536040 & 1.1219910130 & -2.4404005339 & -8.3298984893 \\
\hline & 2 & 1.1869707578 & 1.9774764389 & 1.4492915543 & -1.2424185538 & -7.5536537227 \\
\hline \multirow{3}{*}{$\begin{array}{l}p_{i}=150 \\
p_{0}=300 \\
\theta_{0}=800\end{array}$} & 0 & 8.3454532713 & 3.4064916133 & 0.0677188786 & -3.9596783083 & -8.9602460739 \\
\hline & 1 & 0.3799644417 & 2.7171537078 & 0.8969909208 & -2.6654005810 & -8.5548984885 \\
\hline & 2 & 1.1503024510 & 1.8118357165 & 1.2081310255 & -1.5371690384 & -7.8899735899 \\
\hline \multirow{3}{*}{$\begin{array}{l}p_{i}=300 \\
p_{0}=150 \\
\theta_{0}=800\end{array}$} & 0 & 8.8188658525 & 3.6471597197 & 0.2649871677 & -3.7776107003 & -8.7852160047 \\
\hline & 1 & 3.6049639860 & 2.9421535165 & 1.1219910039 & -2.4404004285 & -8.3298983452 \\
\hline & 2 & 1.1869707577 & 1.9774764387 & 1.4492915543 & -1.2424185537 & -7.5536537224 \\
\hline \multirow{3}{*}{$1 E-4 * T_{\theta \theta} \mathrm{MPa}$} & \multirow{3}{*}{$e_{1}$} & \multicolumn{5}{|c|}{$r$} \\
\hline & & \multicolumn{5}{|c|}{$m=0.4, \omega=500$} \\
\hline & & 0.1 & 0.2 & 0.3 & 0.4 & 0.5 \\
\hline \multirow{3}{*}{$\begin{array}{l}p_{i}=150 \\
p_{0}=300 \\
\theta_{0}=0\end{array}$} & 0 & 1.1199643681 & 0.4548287897 & 0.0269123600 & -0.4863252783 & -1.2191626393 \\
\hline & 1 & 0.6272813080 & 0.5095768873 & 0.1910926579 & -0.4339086008 & -1.5351994013 \\
\hline & 2 & 0.3263905498 & 0.5038039436 & 0.3586769803 & -0.3274307737 & -1.9402396397 \\
\hline \multirow{3}{*}{$\begin{array}{l}p_{i}=300 \\
p_{0}=150 \\
\theta_{0}=0\end{array}$} & 0 & 1.1512903744 & 0.4761073745 & 0.0479630332 & -0.4638575699 & -1.1948611058 \\
\hline & 1 & 0.6497506092 & 0.5320656463 & 0.2136358300 & -0.4115308637 & -1.5126545536 \\
\hline & 2 & 0.3354452568 & 0.5269344433 & 0.3869603568 & -0.3022694774 & -1.9278605585 \\
\hline \multirow{3}{*}{$\begin{array}{l}p_{i}=150 \\
p_{0}=300 \\
\theta_{0}=400\end{array}$} & 0 & 1.1199644787 & 0.4548287386 & 0.0269122948 & -0.4863252189 & -1.2191625155 \\
\hline & 1 & 0.6272812978 & 0.5095769102 & 0.1910926887 & -0.4339085404 & -1.5351993416 \\
\hline & 2 & 0.3263905755 & 0.5038038300 & 0.3586769873 & -0.3274306978 & -1.9402449592 \\
\hline \multirow{3}{*}{$\begin{array}{l}p_{i}=300 \\
p_{0}=150 \\
\theta_{0}=400\end{array}$} & 0 & 1.1512904788 & 0.4761073218 & 0.0479628568 & -0.4638573696 & -1.1948613670 \\
\hline & 1 & 0.6497506075 & 0.5320656332 & 0.2136358153 & -0.4115307488 & -1.5126545365 \\
\hline & 2 & 0.3354452679 & 0.5269344696 & 0.3869603369 & -0.3022695403 & -1.9278600529 \\
\hline \multirow{3}{*}{$\begin{array}{l}p_{i}=150 \\
p_{0}=300 \\
\theta_{0}=800\end{array}$} & 0 & 1.1199642715 & 0.4548288045 & 0.0269123491 & -0.4863251255 & -1.2191624769 \\
\hline & 1 & 1.1199644787 & 0.4548287386 & 0.0269122948 & -0.4863252189 & -1.2191625155 \\
\hline & 2 & 0.3263905007 & 0.5038038190 & 0.3586771087 & -0.3274306558 & -1.9402425453 \\
\hline \multirow{3}{*}{$\begin{array}{l}p_{i}=300 \\
p_{0}=150 \\
\theta_{0}=800\end{array}$} & 0 & 1.1512903765 & 0.4761073125 & 0.0479629909 & -0.4638574341 & -1.1948612858 \\
\hline & 1 & 0.6497505961 & 0.5320656017 & 0.2136358897 & -0.4115308258 & -1.5126545465 \\
\hline & 2 & 0.3354452477 & 0.5269344295 & 0.3869603736 & -0.3022695113 & -1.9278604854 \\
\hline
\end{tabular}

composite cylinder with varying thickness and density as can be seen from Table 4 .

Figures 2-4 have been drawn to discuss the effect of internal and external pressure on stresses in rotating cylinder made of functionally graded stainless steel composite material with constant thickness and constant density with nonlinear strain hardening measure.

It has been observed from Figure 2 that circumferential stress approaches towards compressive from tensile. It has also been observed that when external pressure is greater than 
TABLE 2: Circumferential stresses for rotating cylinder with constant thickness and constant density with $\omega=300,500$, nonlinear strain hardening measure $m=0.6$, and different parameters of Young's modulus under internal and external pressure.

\begin{tabular}{|c|c|c|c|c|c|c|}
\hline \multirow[t]{2}{*}{$1 E-3 * T_{\theta \theta} \mathrm{MPa}$} & \multirow[t]{2}{*}{$e_{1}$} & \multicolumn{5}{|c|}{$\begin{array}{c}r \\
m=0.6, \omega=300\end{array}$} \\
\hline & & 0.1 & 0.2 & 0.3 & 0.4 & 0.5 \\
\hline \multirow{3}{*}{$\begin{array}{l}p_{i}=150 \\
p_{0}=300 \\
\theta_{0}=0\end{array}$} & 0 & 8.3453827289 & 3.4064900899 & 0.0677286065 & -3.9596681544 & -8.9602414309 \\
\hline & 1 & 3.3799456580 & 2.7171435351 & 0.8969901053 & -2.6653896254 & -8.5548804228 \\
\hline & 2 & 1.1502969495 & 1.8118279175 & 1.2081251826 & -1.5371621151 & -7.8899506975 \\
\hline \multirow{3}{*}{$\begin{array}{l}p_{i}=300 \\
p_{0}=150 \\
\theta_{0}=0\end{array}$} & 0 & 8.8187936296 & 3.6471591317 & 0.2649975386 & -3.7776011860 & -8.7852121667 \\
\hline & 1 & 3.6049445586 & 2.9421431766 & 1.1219899984 & -2.4403890591 & -8.3298798957 \\
\hline & 2 & 1.1869649855 & 1.9774683014 & 1.4492853591 & -1.2424112423 & -7.5536293888 \\
\hline \multirow{3}{*}{$\begin{array}{l}p_{i}=150 \\
p_{0}=300 \\
\theta_{0}=400\end{array}$} & 0 & 8.3453833202 & 3.4064903276 & 0.0677284767 & -3.9596684114 & -8.9602417913 \\
\hline & 1 & 3.3799454509 & 2.7171434189 & 0.8969901711 & -2.6653895895 & -8.5548805034 \\
\hline & 2 & 1.1502969501 & 1.8118279178 & 1.2081251821 & -1.5371621158 & -7.8899507229 \\
\hline \multirow{3}{*}{$\begin{array}{l}p_{i}=300 \\
p_{0}=150 \\
\theta_{0}=400\end{array}$} & 0 & 8.8187922056 & 3.6471584895 & 0.2649978782 & -3.7776012451 & -8.7852123681 \\
\hline & 1 & 3.6049444470 & 2.9421430233 & 1.1219900447 & -2.4403890386 & -8.3298802292 \\
\hline & 2 & 1.1869649854 & 1.9774683013 & 1.4492853591 & -1.2424112422 & -7.5536293885 \\
\hline \multirow{3}{*}{$\begin{array}{l}p_{i}=150 \\
p_{0}=300 \\
\theta_{0}=800\end{array}$} & 0 & 8.3453820024 & 3.4064896339 & 0.0677286501 & -3.9596681965 & -8.9602415728 \\
\hline & 1 & 3.3799459533 & 2.7171437210 & 0.8969899701 & -2.6653898309 & -8.5548807795 \\
\hline & 2 & 1.1502969490 & 1.8118279176 & 1.2081251818 & -1.5371621155 & -7.8899507012 \\
\hline \multirow{3}{*}{$\begin{array}{l}p_{i}=300 \\
p_{0}=150 \\
\theta_{0}=800\end{array}$} & 0 & 8.8187929120 & 3.6471586248 & 0.2649979578 & -3.7776012681 & -8.7852105705 \\
\hline & 1 & 3.6049446223 & 2.9421429798 & 1.1219900968 & -2.4403889573 & -8.3298789707 \\
\hline & 2 & 1.1869649853 & 1.9774683011 & 1.4492853590 & -1.2424112421 & -7.5536293882 \\
\hline \multirow{3}{*}{$1 E-4 * T_{\theta \theta} \mathrm{MPa}$} & \multirow{3}{*}{$e_{1}$} & & & $r$ & & \\
\hline & & & & $m=0.6, \omega=50$ & & \\
\hline & & 0.1 & 0.2 & 0.3 & 0.4 & 0.5 \\
\hline \multirow{3}{*}{$\begin{array}{l}p_{i}=150 \\
p_{0}=300 \\
\theta_{0}=0\end{array}$} & 0 & 1.1199583152 & 0.4548258695 & 0.0269146492 & -0.4863265526 & -1.2191654300 \\
\hline & 1 & 0.6272799179 & 0.5095770638 & 0.1910931884 & -0.4339078950 & -1.5352039429 \\
\hline & 2 & 0.3263903739 & 0.5038038750 & 0.3586772209 & -0.3274306376 & -1.9402344736 \\
\hline \multirow{3}{*}{$\begin{array}{l}p_{i}=300 \\
p_{0}=150 \\
\theta_{0}=0\end{array}$} & 0 & 1.1512567236 & 0.4761058146 & 0.0480074980 & -0.4638915755 & -1.1948352560 \\
\hline & 1 & 0.6496608008 & 0.5320325649 & 0.2137603620 & -0.4118738110 & -1.5125508783 \\
\hline & 2 & 0.3354423760 & 0.5269311856 & 0.3869645881 & -0.3022635649 & -1.9278571554 \\
\hline \multirow{3}{*}{$\begin{array}{l}p_{i}=150 \\
p_{0}=300 \\
\theta_{0}=400\end{array}$} & 0 & 1.1199579631 & 0.4548258318 & 0.0269147731 & -0.4863261541 & -1.2191655802 \\
\hline & 1 & 0.6272799298 & 0.5095770743 & 0.1910931873 & -0.4339077941 & -1.5352040493 \\
\hline & 2 & 0.3263904649 & 0.5038039194 & 0.3586771853 & -0.3274308913 & -1.9402307798 \\
\hline \multirow{3}{*}{$\begin{array}{l}p_{i}=300 \\
p_{0}=150 \\
\theta_{0}=400\end{array}$} & 0 & 1.1512569402 & 0.4761057447 & 0.0480074391 & -0.4638917626 & -1.1948352393 \\
\hline & 1 & 0.6496607027 & 0.5320325183 & 0.2137605230 & -0.4118740006 & -1.5125508832 \\
\hline & 2 & 0.3354423676 & 0.5269312306 & 0.3869645451 & -0.3022636353 & -1.9278567951 \\
\hline \multirow{3}{*}{$\begin{array}{l}p_{i}=150 \\
p_{0}=300 \\
\theta_{0}=800\end{array}$} & 0 & 1.1199579102 & 0.4548258849 & 0.0269148341 & -0.4863259137 & -1.2191656124 \\
\hline & 1 & 0.6272799292 & 0.5095770557 & 0.1910931905 & -0.4339078242 & -1.5352038916 \\
\hline & 2 & 0.3263903269 & 0.5038037303 & 0.3586773178 & -0.3274301493 & -1.9402403280 \\
\hline \multirow{3}{*}{$\begin{array}{l}p_{i}=300 \\
p_{0}=150 \\
\theta_{0}=800\end{array}$} & 0 & 1.1512563866 & 0.4761057469 & 0.0480078293 & -0.4638922712 & -1.1948350571 \\
\hline & 1 & 0.6496607065 & 0.5320325064 & 0.2137604900 & -0.4118740845 & -1.5125507865 \\
\hline & 2 & 0.3354423734 & 0.5269312334 & 0.3869645540 & -0.3022636247 & -1.9278571251 \\
\hline
\end{tabular}

the internal pressure, these stresses are maximum at external surface for homogeneous as well as functionally graded stainless steel composite cylinder. Also, it has been observed that circumferential stress is maximum at internal surface for homogeneous cylinder while maximum at external surface for functionally graded stainless steel composite cylinder when external pressure is less than the internal pressure. Also, with the increase in angular speed, circumferential stresses increase significantly. From Figure 3 it can be seen that, as circumferential stresses increase for homogeneous cylinder 
TABLE 3: Circumferential stresses for rotating cylinder with variable thickness and variable density $l=0.7, d=0.5, \omega=300,500$, strain hardening measure $m=0.4$, and different parameters of Young's modulus under internal and external pressure.

\begin{tabular}{|c|c|c|c|c|c|c|}
\hline \multirow[t]{2}{*}{$1 E-3 * T_{\theta \theta} \mathrm{MPa}$} & \multirow[t]{2}{*}{$e_{1}$} & \multicolumn{5}{|c|}{$r$} \\
\hline & & 0.1 & 0.2 & 0.3 & 0.4 & 0.5 \\
\hline \multirow{3}{*}{$\begin{array}{l}p_{i}=150 \\
p_{0}=300 \\
\theta_{0}=0\end{array}$} & 0 & 4.078610121 & 1.692083635 & -0.362927882 & -3.599668076 & -8.388122376 \\
\hline & 1 & 1.844023846 & 1.536347566 & 0.332616455 & -2.596999032 & -8.186025126 \\
\hline & 2 & 0.698588556 & 1.166718627 & 0.723851443 & -1.632455674 & -7.703313292 \\
\hline \multirow{3}{*}{$\begin{array}{l}p_{i}=300 \\
p_{0}=150 \\
\theta_{0}=0\end{array}$} & 0 & 4.468498659 & 1.869943306 & -0.216047724 & -3.455691317 & -8.239259816 \\
\hline & 1 & 2.039946535 & 1.721858320 & 0.520942472 & -2.403128817 & -7.985940189 \\
\hline & 2 & 0.725452105 & 1.314126561 & 0.957769537 & -1.332711442 & -7.351503187 \\
\hline \multirow{3}{*}{$\begin{array}{l}p_{i}=150 \\
p_{0}=300 \\
\theta_{0}=400\end{array}$} & 0 & 4.078610926 & 1.692083796 & -0.362928200 & -3.599667939 & -8.388124156 \\
\hline & 1 & 1.844023759 & 1.536347360 & 0.332616655 & -2.596998868 & -8.186026405 \\
\hline & 2 & 0.698588485 & 1.166718653 & 0.723851463 & -1.632455697 & -7.703312785 \\
\hline \multirow{3}{*}{$\begin{array}{l}p_{i}=300 \\
p_{0}=150 \\
\theta_{0}=400\end{array}$} & 0 & 4.468498329 & 1.869943517 & -0.216047300 & -3.455691093 & -8.239251730 \\
\hline & 1 & 2.039946605 & 1.721858238 & 0.520942423 & -2.403128796 & -7.985940383 \\
\hline & 2 & 0.725452128 & 1.314126566 & 0.957769488 & -1.332711458 & -7.351503426 \\
\hline \multirow{3}{*}{$\begin{array}{l}p_{i}=150 \\
p_{0}=300 \\
\theta_{0}=800\end{array}$} & 0 & 4.078610278 & 1.692083456 & -0.362927548 & -3.599667594 & -8.388125027 \\
\hline & 1 & 1.844023766 & 1.536347565 & 0.332616556 & -2.596998868 & -8.186026291 \\
\hline & 2 & 0.698588473 & 1.166718654 & 0.723851445 & -1.632455676 & -7.703312834 \\
\hline \multirow{3}{*}{$\begin{array}{l}p_{i}=300 \\
p_{0}=150 \\
\theta_{0}=800\end{array}$} & 0 & 4.468498296 & 1.869943321 & -0.216047655 & -3.455691489 & -8.239237723 \\
\hline & 1 & 2.039946200 & 1.721858288 & 0.520942491 & -2.403128819 & -7.985940064 \\
\hline & 2 & 0.725452082 & 1.314126521 & 0.957769539 & -1.332711402 & -7.351503219 \\
\hline
\end{tabular}

$1 E-4 * T_{\theta \theta} \mathrm{MPa} \quad e_{1} \quad m=0.4, \omega=500, l=0.7, d=0.5$

\begin{tabular}{llllrrrr} 
& & 0.1 & 0.2 & 0.3 & 0.4 & 0.5 \\
\hline$p_{i}=150$, & 0 & 0.502344054 & 0.209118575 & -0.032085608 & -0.411840852 & -1.075755914 \\
$p_{0}=300$, & 1 & 0.301690685 & 0.253918828 & 0.067872877 & -0.387759274 & -1.337560991 \\
$\theta_{0}=0$ & 2 & 0.170338387 & 0.272622555 & 0.177093634 & -0.328795174 & -1.672186161 \\
$p_{i}=300$, & 0 & 0.520602572 & 0.223194821 & -0.013327208 & -0.385714307 & -1.041663604 \\
$p_{0}=150$, & 1 & 0.314858338 & 0.269151250 & 0.087139893 & -0.362225697 & -1.303362831 \\
$\theta_{0}=0$ & 2 & 0.175533196 & 0.289517104 & 0.201213547 & -0.301959686 & -1.648231058 \\
$p_{i}=150$, & 0 & 0.502344076 & 0.209118532 & -0.032085631 & -0.411840853 & -1.075756093 \\
$p_{0}=300$, & 1 & 0.301690546 & 0.253918731 & 0.067873081 & -0.387757210 & -1.337562051 \\
$\theta_{0}=400$ & 2 & 0.170338398 & 0.272622541 & 0.177093558 & -0.328795106 & -1.672185933 \\
$p_{i}=300$, & 0 & 0.520602619 & 0.223194813 & -0.013327161 & -0.385714149 & -1.041664533 \\
$p_{0}=150$, & 1 & 0.314858326 & 0.269151199 & 0.087139890 & -0.362225687 & -1.303362773 \\
$\theta_{0}=400$ & 2 & 0.175533191 & 0.289517093 & 0.201213591 & -0.301959717 & -1.648231090 \\
$p_{i}=150$, & 0 & 0.502344041 & 0.209118525 & -0.032085593 & -0.411841019 & -1.075755756 \\
$p_{0}=300$, & 1 & 0.301690548 & 0.253918749 & 0.067873075 & -0.387757974 & -1.337560151 \\
$\theta_{0}=800$ & 2 & 0.170338413 & 0.272622528 & 0.177093598 & -0.328795120 & -1.672185805 \\
$p_{i}=300$, & 0 & 0.520602532 & 0.223194802 & -0.013327191 & -0.385714162 & -1.041664483 \\
$p_{0}=150$, & 1 & 0.314858370 & 0.269151215 & 0.087139921 & -0.362225740 & -1.303362723 \\
$\theta_{0}=800$ & 2 & 0.175533168 & 0.289517159 & 0.201213488 & -0.301959669 & -1.648230944 \\
\hline
\end{tabular}

as well as for nonhomogenous cylinder but with the change in nonhomogeneity from $e_{1}=1$ to $e_{1}=2$, circumferential stresses decrease when external pressure is greater than the internal pressure with thermal effects. Also, these stresses increase for homogenous cylinder as well as for functionally graded stainless steel composite cylinder $\left(e_{1}=1\right)$ while decrease for functionally graded stainless steel composite cylinder with $e_{1}=2$. With the increase in temperature these 
TABLE 4: Circumferential stresses for rotating cylinder with variable thickness and variable density $l=0.7, d=0.5, \omega=300,500$, strain hardening measure $m=0.6$, and different parameters of Young's modulus under internal and external pressure.

\begin{tabular}{|c|c|c|c|c|c|c|}
\hline \multirow[t]{2}{*}{$1 E-3 * T_{\theta \theta} \mathrm{MPa}$} & \multirow[t]{2}{*}{$e_{1}$} & \multicolumn{5}{|c|}{$\begin{array}{c}r \\
m=0.6, \omega=300, l=0.7, d=0.5\end{array}$} \\
\hline & & 0.1 & 0.2 & 0.3 & 0.4 & 0.5 \\
\hline \multirow{3}{*}{$\begin{array}{l}p_{i}=150 \\
p_{0}=300 \\
\theta_{0}=0\end{array}$} & 0 & 4.078550787 & 1.692092827 & -0.362917634 & -3.599662505 & -8.388124303 \\
\hline & 1 & 1.844008854 & 1.536344037 & 0.332619755 & -2.596989139 & -8.186011860 \\
\hline & 2 & 0.698583918 & 1.166713498 & 0.723849363 & -1.632447308 & -7.703291360 \\
\hline \multirow{3}{*}{$\begin{array}{l}p_{i}=300 \\
p_{0}=150 \\
\theta_{0}=0\end{array}$} & 0 & 4.468438868 & 1.869957168 & -0.216034432 & -3.455686049 & -8.239492345 \\
\hline & 1 & 2.039930194 & 1.721854313 & 0.520946730 & -2.403118071 & -7.985927195 \\
\hline & 2 & 0.725447053 & 1.314120776 & 0.957767141 & -1.332701862 & -7.351479020 \\
\hline \multirow{3}{*}{$\begin{array}{l}p_{i}=150 \\
p_{0}=300 \\
\theta_{0}=400\end{array}$} & 0 & 4.078550758 & 1.692093003 & -0.362917541 & -3.599662684 & -8.388124758 \\
\hline & 1 & 1.844009034 & 1.536344170 & 0.332619845 & -2.596989055 & -8.186013323 \\
\hline & 2 & 0.698583989 & 1.166713507 & 0.723849336 & -1.632447294 & -7.703291352 \\
\hline \multirow{3}{*}{$\begin{array}{l}p_{i}=300 \\
p_{0}=150 \\
\theta_{0}=400\end{array}$} & 0 & 4.468440841 & 1.869957567 & -0.216033626 & -3.455685275 & -8.239588040 \\
\hline & 1 & 2.039930222 & 1.721854440 & 0.520946513 & -2.403117933 & -7.985926001 \\
\hline & 2 & 0.725447107 & 1.314120882 & 0.957767050 & -1.332701999 & -7.351479230 \\
\hline \multirow{3}{*}{$\begin{array}{l}p_{i}=150 \\
p_{0}=300 \\
\theta_{0}=800\end{array}$} & 0 & 4.078551089 & 1.692092847 & -0.362917694 & -3.599662435 & -8.388126479 \\
\hline & 1 & 1.844008698 & 1.536343957 & 0.332619859 & -2.596988916 & -8.186012239 \\
\hline & 2 & 0.698583989 & 1.166713524 & 0.723849328 & -1.632447317 & -7.703291542 \\
\hline \multirow{3}{*}{$\begin{array}{l}p_{i}=300 \\
p_{0}=150 \\
\theta_{0}=800\end{array}$} & 0 & 4.468438120 & 1.869956228 & -0.216035341 & -3.455687051 & -8.239368632 \\
\hline & 1 & 2.039930039 & 1.721854569 & 0.520946538 & -2.403117966 & -7.985925731 \\
\hline & 2 & 0.725447049 & 1.314120768 & 0.957767137 & -1.332701833 & -7.351479029 \\
\hline
\end{tabular}

$1 E-4 * T_{\theta \theta} \mathrm{MPa} \quad e_{1} \quad m=0.6, \omega=500, l=0.7, d=0.5$

\begin{tabular}{llcccrrr} 
& & 0.1 & 0.2 & 0.3 & 0.4 & 0.5 \\
\hline$p_{i}=150$, & 0 & 0.502339190 & 0.209119023 & -0.032080434 & -0.411843003 & -1.075751575 \\
$p_{0}=300$, & 1 & 0.301689441 & 0.253919137 & 0.067872897 & -0.387758158 & -1.337567443 \\
$\theta_{0}=0$ & 2 & 0.170338021 & 0.272622433 & 0.177094183 & -0.328793821 & -1.672191050 \\
$p_{i}=300$, & 0 & 0.520600260 & 0.223196743 & -0.013328691 & -0.385729358 & -1.041635950 \\
$p_{0}=150$, & 1 & 0.314857828 & 0.269151955 & 0.087140006 & -0.362234515 & -1.303350674 \\
$\theta_{0}=0$ & 2 & 0.175532879 & 0.289517002 & 0.201213980 & -0.301960040 & -1.648233514 \\
$p_{i}=150$, & 0 & 0.502339173 & 0.209119009 & -0.032080335 & -0.411843048 & -1.075752013 \\
$p_{0}=300$, & 1 & 0.301689029 & 0.253918998 & 0.067873493 & -0.387754850 & -1.337566825 \\
$\theta_{0}=400$ & 2 & 0.170338061 & 0.272622405 & 0.177094107 & -0.328793799 & -1.672190560 \\
$p_{i}=300$, & 0 & 0.520600268 & 0.223196732 & -0.013328653 & -0.385729330 & -1.041636334 \\
$p_{0}=150$, & 1 & 0.314857844 & 0.269151936 & 0.087139999 & -0.362234465 & -1.303350722 \\
$\theta_{0}=400$ & 2 & 0.175532856 & 0.289516980 & 0.201214076 & -0.301960031 & -1.648233768 \\
$p_{i}=150$, & 0 & 0.502339102 & 0.209118939 & -0.032080279 & -0.411842835 & -1.075752159 \\
$p_{0}=300$, & 1 & 0.301689252 & 0.253919092 & 0.067873202 & -0.387759066 & -1.337565663 \\
$\theta_{0}=800$ & 2 & 0.170338011 & 0.272622432 & 0.177094145 & -0.328793808 & -1.672190610 \\
$p_{i}=300$, & 0 & 0.520600345 & 0.223196689 & -0.013328705 & -0.385729338 & -1.041635876 \\
$p_{0}=150$, & 1 & 0.314857846 & 0.269151949 & 0.087139963 & -0.362234522 & -1.303350759 \\
$\theta_{0}=800$ & 2 & 0.175532843 & 0.289516995 & 0.201214043 & -0.301960031 & -1.648233661 \\
\hline
\end{tabular}

stresses decrease significantly for homogeneous as well as for functionally graded stainless steel composite cylinder as can be seen from Figure 4.
Figures 5-7 have been drawn to discuss the effect of internal and external pressure on stresses in rotating cylinder made of functionally graded stainless steel composite 


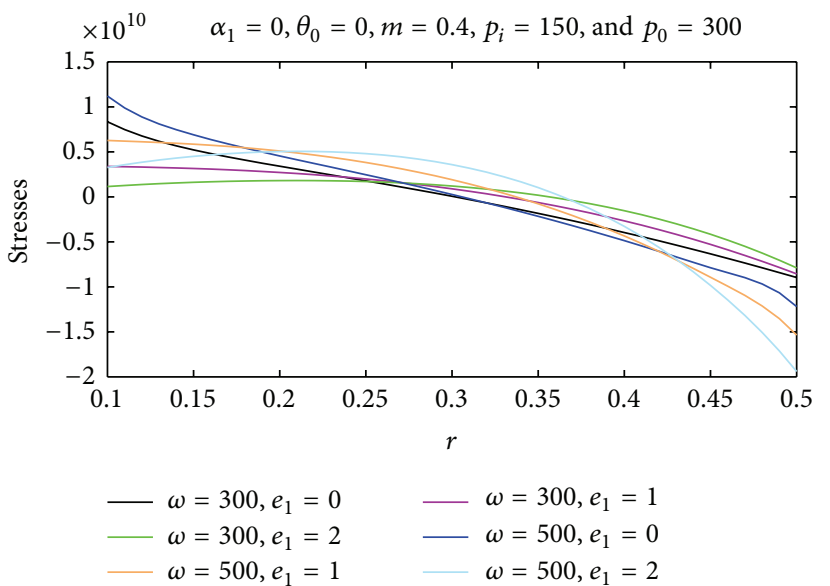

(a)

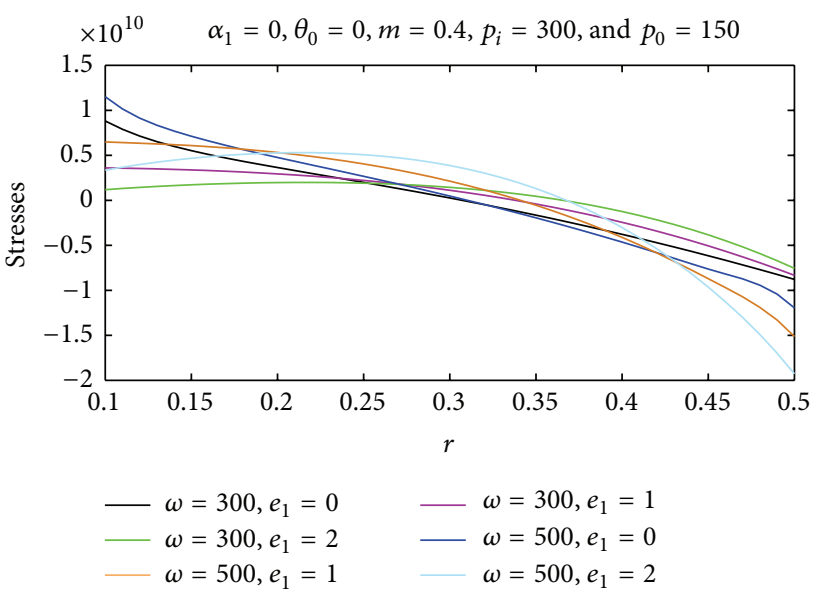

(b)

FIGURE 2: Elastic-plastic stresses in a rotating cylinder with constant thickness and constant density without thermal effects $\left(\theta_{0}=0\right)$ with parameters $e_{1}=0,1,2$ and strain hardening measure $m=0.4$ under internal and external pressure.

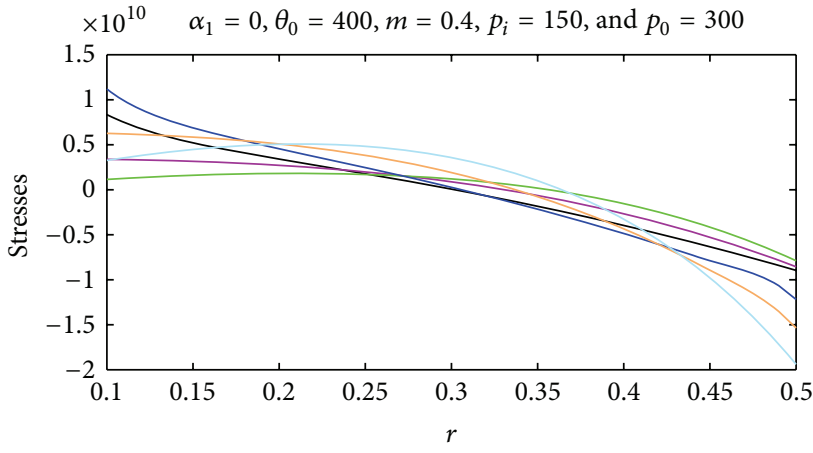

(a)

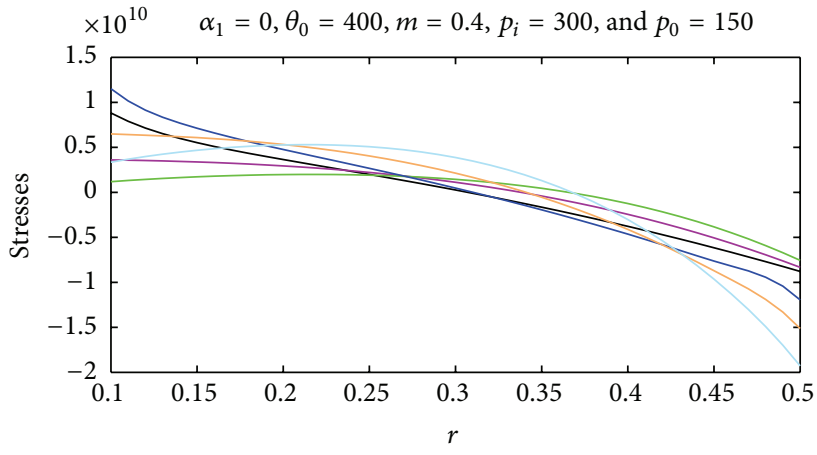

(b)

FIGURE 3: Elastic-plastic stresses in a rotating cylinder with constant thickness and constant density with thermal effects $\left(\theta_{0}=400\right)$ with parameters $e_{1}=0,1,2$ and strain hardening measure $m=0.4$ under internal and external pressure.

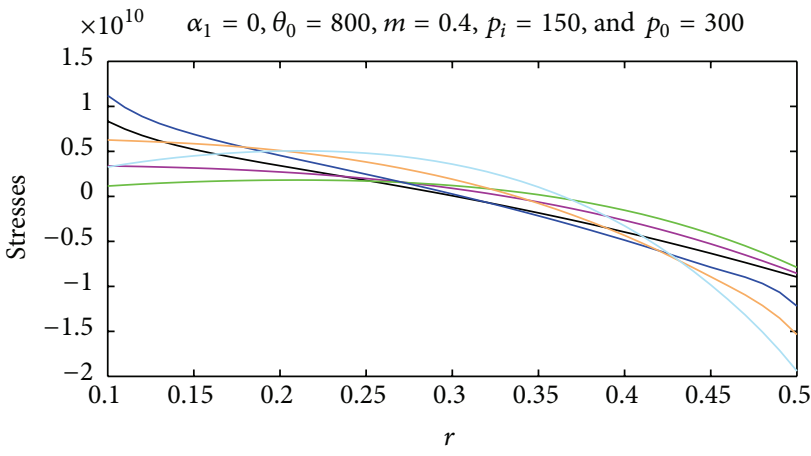

(a)

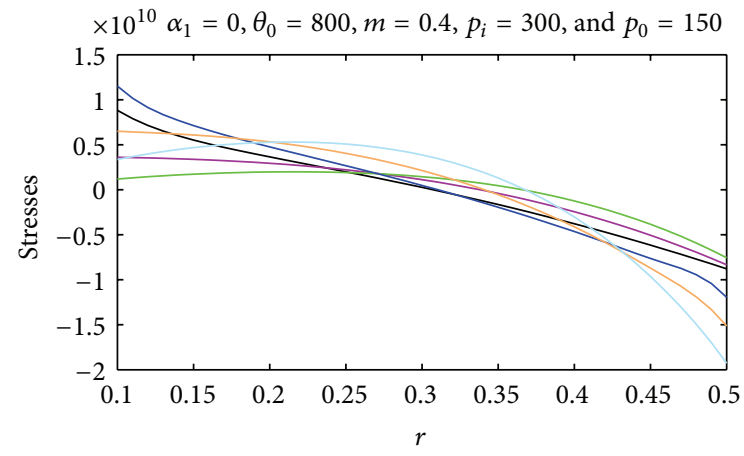

(b)

FIGURE 4: Elastic-plastic stresses in a rotating cylinder with constant thickness and constant density with thermal effects $\left(\theta_{0}=800\right)$ with parameters $e_{1}=0,1,2$ and strain hardening measure $m=0.4$ under internal and external pressure. 


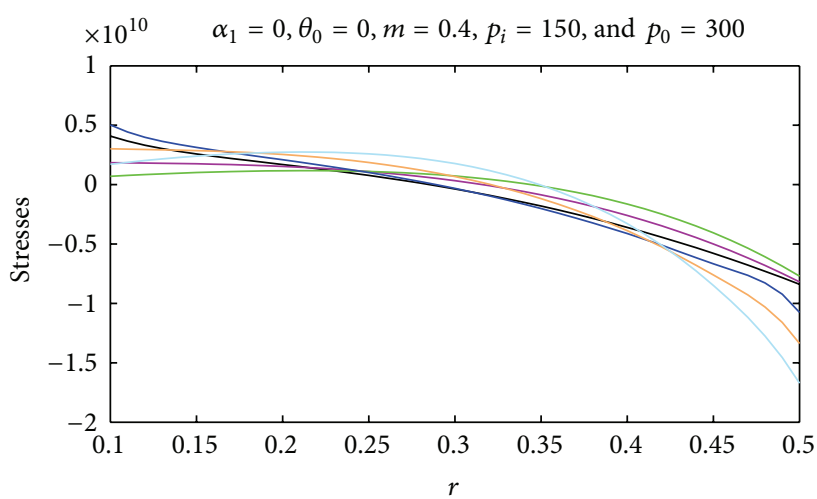

(a)

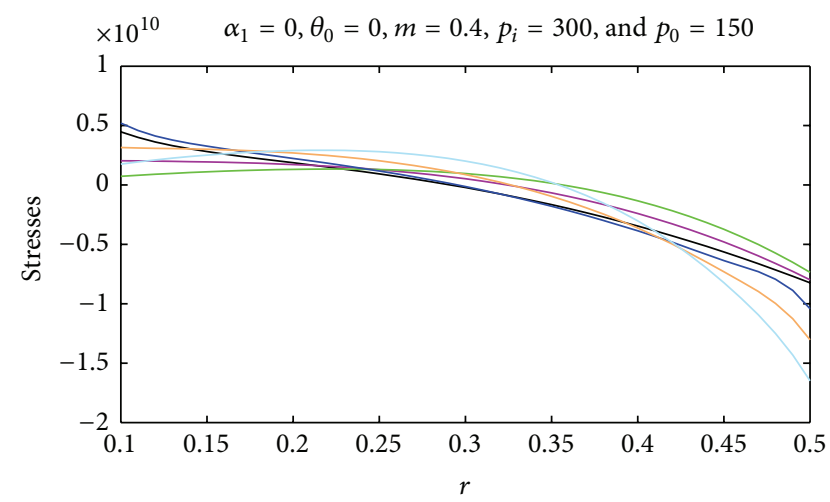

(b)

FIGURE 5: Elastic-plastic stresses in a rotating cylinder with variable thickness and variable density without thermal effects $\left(\theta_{0}=0\right)$ with parameters $e_{1}=0,1,2$ and strain hardening measure $m=0.4$ under internal and external pressure.

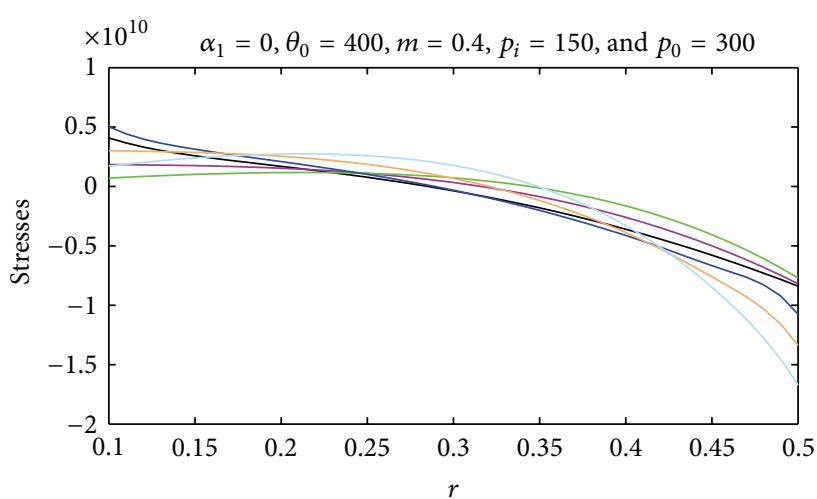

(a)

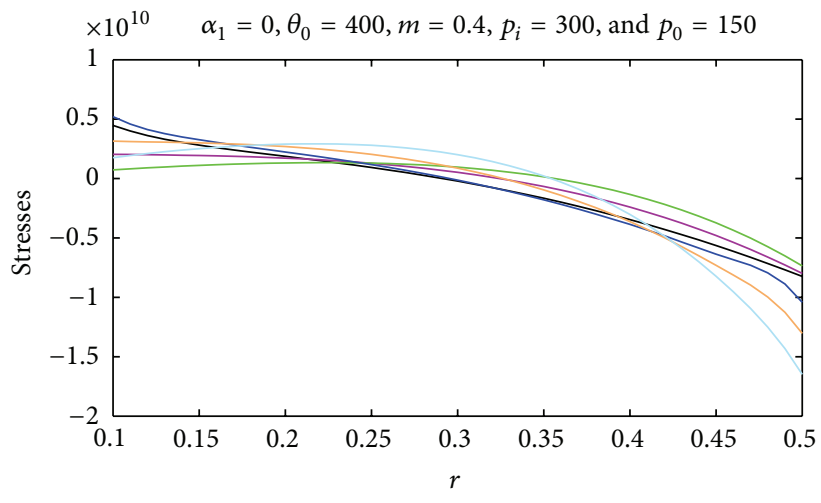

(b)

FIGURE 6: Elastic-plastic stresses in a rotating cylinder with variable thickness and variable density with thermal effects $\left(\theta_{0}=400\right)$ with parameters $e_{1}=0,1,2$ and strain hardening measure $m=0.4$ under internal and external pressure.

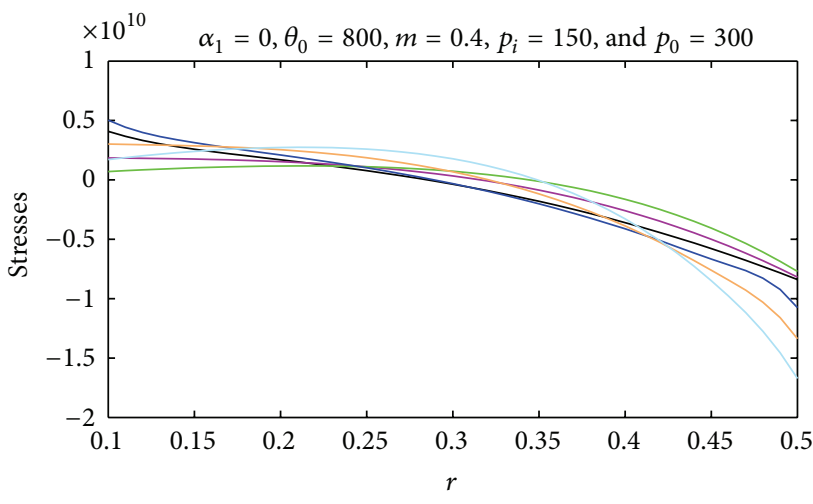

(a)

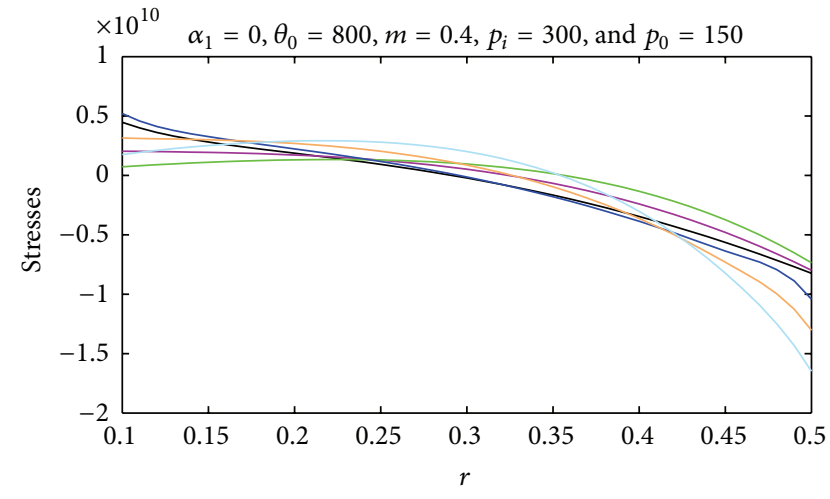

(b)

FIGURE 7: Elastic-plastic stresses in a rotating cylinder with variable thickness and variable density with thermal effects $\left(\theta_{0}=800\right)$ with parameters $e_{1}=0,1,2$ and strain hardening measure $m=0.4$ under internal and external pressure. 
material with variable thickness and variable density with nonlinear strain hardening measure.

It has been observed from Figure 5 that circumferential stresses are maximum at external surface for homogeneous as well as functionally graded stainless steel composite cylinder. It has also been observed that with the increase in angular speed circumferential stresses increase significantly. With the introduction of thermal effects, circumferential stresses increase for homogeneous cylinder as well as for functionally graded stainless steel composite cylinder $\left(e_{1}=\right.$ 1 ), but with the change in nonhomogeneity from $e_{1}=1$ to $e_{1}=2$, circumferential stresses decrease when external pressure is greater than the internal pressure as can be seen from Figure 6. Circumferential stresses decrease for homogeneous cylinder while increase for functionally graded stainless steel composite cylinder when external pressure is less than the internal pressure. It has also been observed from Figure 7 that with the increase in temperature these stresses increase significantly for homogeneous cylinder as well for functionally graded stainless steel composite cylinder with $e_{1}=1$ while decrease for functionally graded stainless steel composite cylinder with $e_{1}=2$ when external pressure is greater than the internal pressure, while these stresses decrease significantly for homogeneous cylinder as well for functionally graded stainless steel composite cylinder when external pressure is less than the internal pressure.

\section{Conclusion}

From the analysis, we can conclude that rotating cylinder made of functionally graded stainless steel composite material having variable thickness and variable density with Swift's strain hardening measure $m=0.6$ and thermal loading is better choice for designers as compared to rotating cylinder with constant thickness and constant density. This is because of the reason that circumferential stress is less for functionally graded stainless steel composite cylinder with variable thickness and variable density as compared to other cases, which leads to the idea of stress saving that minimizes the possibility of fracture of cylinder.

\section{Conflict of Interests}

The authors declare that they have no conflict of interest.

\section{References}

[1] S. Suresh and A. Mortensen, Fundamentals of Functionally Graded Materials, IOM ${ }^{3}$, Maney Publishing, London, UK, 1998.

[2] J. N. Reddy, "Analysis of functionally graded plates," International Journal for Numerical Methods in Engineering, vol. 47, no. 1-3, pp. 663-684, 2000.

[3] S. P. Timoshenko and J. N. Goodier, Theory of Elasticity, McGraw-Hill, NewYork, NY, USA, 3rd edition, 1970.

[4] R. Hill, The Mathematical Theory of Plasticity, Oxford University Press, Oxford, UK, 1998.

[5] Y. Obata, T. Oji, and N. Noda, "Steady thermal stresses in a hollow circular cylinder and a hollow sphere made of functionally graded material (analysis with perturbation method)," Reports of the National Industrial Research Institute of Nagoya, vol. 47, no. 4, pp. 317-338, 1998.

[6] J. Perry and J. Aboudi, "Elasto-plastic stresses in thick walled cylinders," Journal of Pressure Vessel Technology, vol. 125, no. 3, pp. 248-252, 2003.

[7] X.-L. Gao, "Elasto-plastic analysis of an internally pressurized thick-walled cylinder using a strain gradient plasticity theory," International Journal of Solids and Structures, vol. 40, no. 23, pp. 6445-6455, 2003.

[8] N. Tutuncu and M. Ozturk, "Exact solutions for stresses in functionally graded pressure vessels," Composites $B$, vol. 32, no. 8, pp. 683-686, 2001.

[9] T. Singh and V. K. Gupta, "Creep analysis of an internally pressurized thick cylinder made of a functionally graded composite," Journal of Strain Analysis for Engineering Design, vol. 44, no. 7, pp. 583-594, 2009.

[10] A. K. Aggarwal, Sharma, Richa, and Sharma Sanjeev, "Safety analysis using lebesgue strain measure of thick-walled cylinder for functionally graded material under internal and external pressure," The Scientific World Journal, vol. 2013, Article ID 676190, 10 pages, 2013.

[11] A. K. Aggarwal, S. Richa, and S. Sanjeev, "Safety analysis of thermal creep non-homogeneous thick-walled circular cylinder under internal and external pressure using lebesgue strain measure," Multidiscipline Modeling in Materials and Structures, vol. 9, no. 4, 2013.

[12] A. Parvizi, R. Naghdabadi, and J. Arghavani, "Analysis of Al A359/SiCp functionally graded cylinder subjected to internal pressure and temperature gradient with elastic-plastic deformation," Journal of Thermal Stresses, vol. 34, no. 10, pp. 1054-1070, 2011.

[13] A. N. Eraslan and F. Akgül, "Yielding and elastoplastic deformation of annular disks of a parabolic section subject to external compression," Turkish Journal of Engineering and Environmental Sciences, vol. 29, no. 1, pp. 51-60, 2005. 

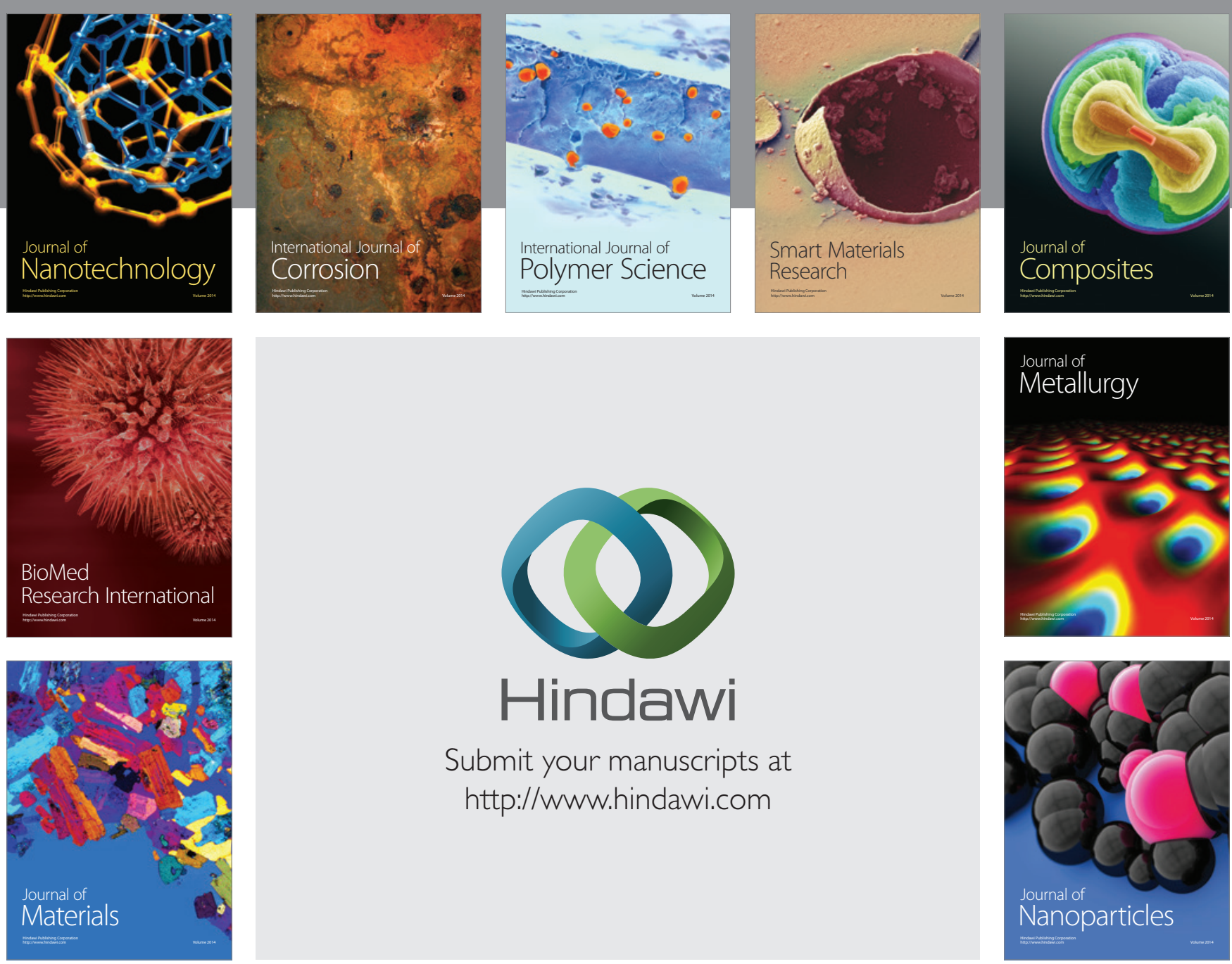

Submit your manuscripts at http://www.hindawi.com
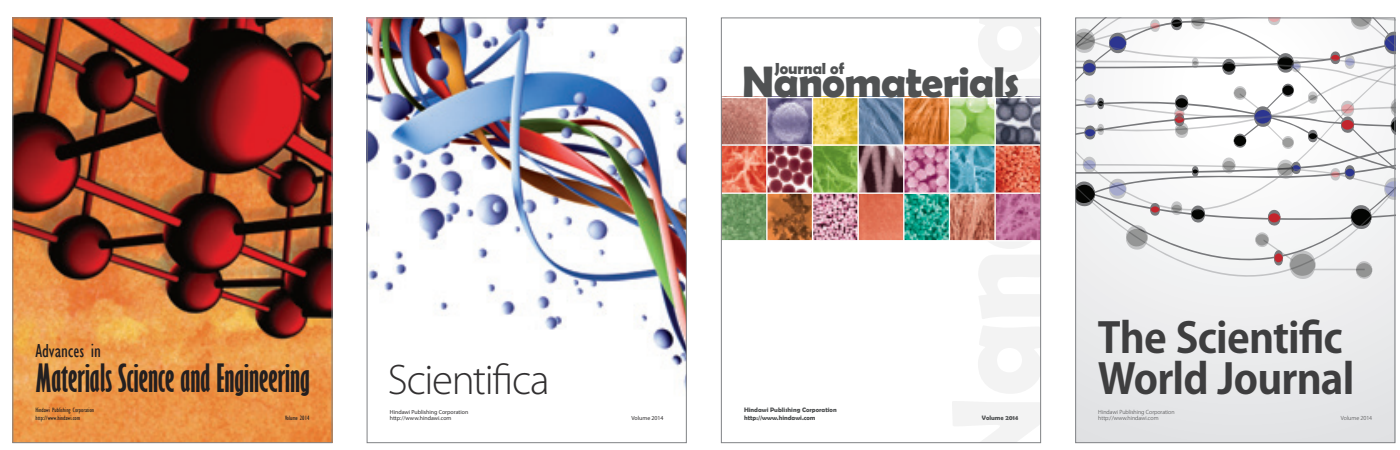

\section{The Scientific World Journal}
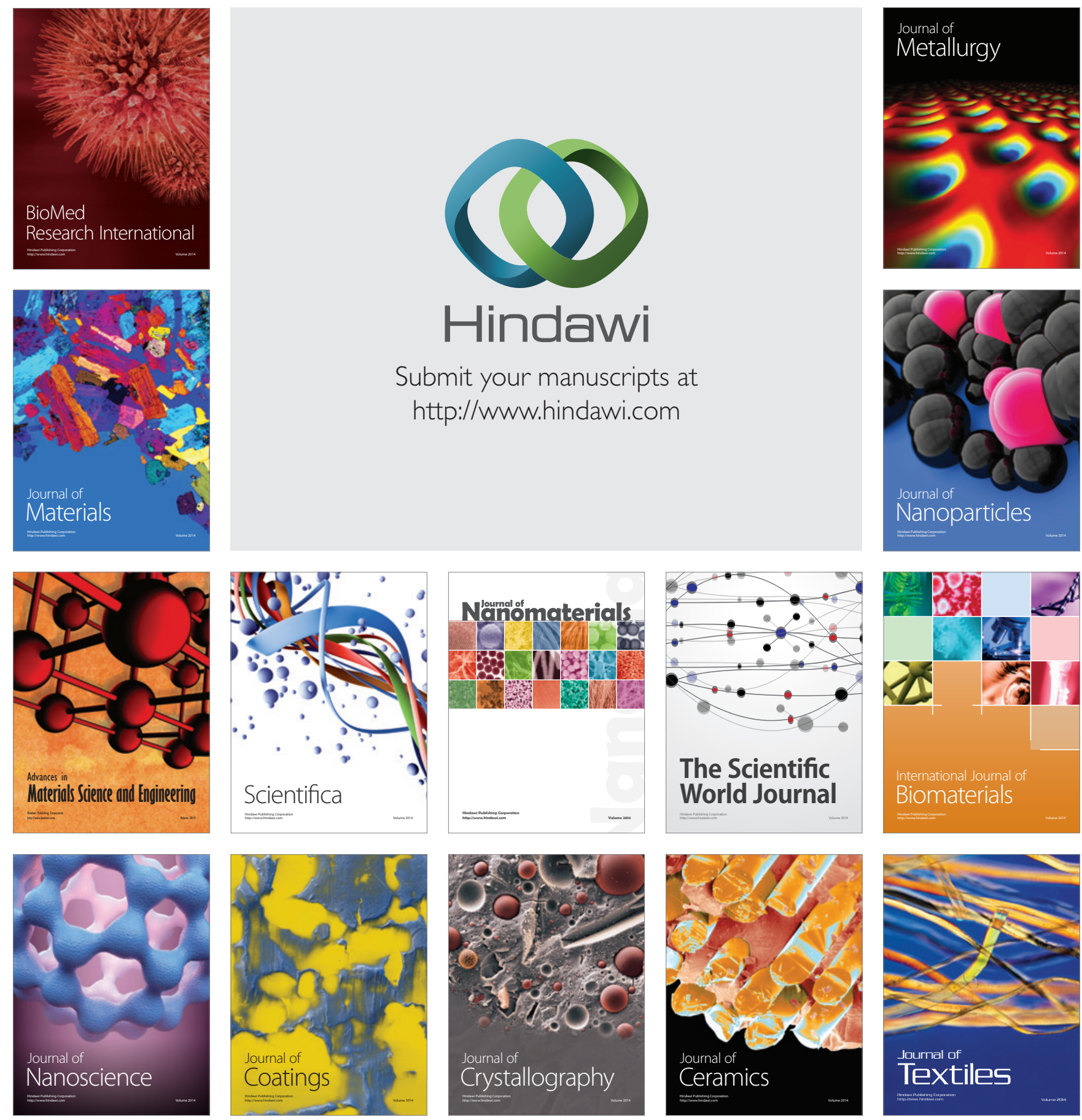